\title{
Substrate Integrated Waveguide (SIW) with Koch Fractal Electromagnetic Bandgap Structures (KFEBG) for Bandpass Filter Design
}

\author{
Juan de Dios Ruiz, Félix L. Martínez-Viviente, Alejandro Álvarez-Melcón and Juan Hinojosa
}

\begin{abstract}
High-performance bandpass filters (BPFs) based on substrate integrated waveguide (SIW) and half mode SIW (HMSIW) with Koch fractal electromagnetic bandgap (KFEBG) patterns etched on the waveguide surface are proposed. These BPFs are designed using a new methodology. The BPF responses are improved by applying a chirped and tapered technique to the KFEBG patterns, which exhibit a radius-to-period ratio $r / a \geq 0.5$. Simulation results and experimental verification are presented. Low insertion loss and high rejection are achieved.
\end{abstract}

Index Terms-Bandpass filters, electromagnetic bandgap (EBG), Koch fractal, substrate integrated waveguide (SIW).

\section{INTRODUCTION}

$\mathrm{S}_{\mathrm{s}}^{\mathrm{u}}$ UBSTRATE integrated waveguide (SIW) and half mode SIW (HMSIW) have been used to develop bandpass filters (BPFs) with electromagnetic bandgap (EBG) patterns [1], [2]. SIW (HMSIW) performances, such as insertion loss, quality factor and power handling capability, are better than those of filters based on conventional microstrip and coplanar waveguides. SIW technology is low cost and has easy integration with other planar circuits. Bandpass responses are obtained by combining the high-pass characteristics of the SIW (HMSIW) [3], [4] and the stopband behavior of EBG patterns. These BPFs present an excellent rejection level due to the periodic EBG patterns, but as a drawback they exhibit reduced width of the stopband, large size, and ripple in the passband. To improve these features, as required by modern microwave systems, we have applied a chirping and tapering technique to Koch fractal EBG (KFEBG) patterns with a radius-to-period ratio $r / a \geq 0.5$ [5], [6]. Fig. 1 shows the layouts of the proposed bandpass filters. They are made of a SIW (HMSIW) waveguide with KFEBG patterns etched on the top plane of the SIW (or on the bottom plane of the HMSIW) and a transition to a $50 \Omega$ microstrip. These SIW (HMSIW)-KFEBG. BPFs have been designed by using an original procedure. Remarkable performances have been obtained.

Manuscript received ??, 2014. This work was supported by the Spanish Ministerio de Economía y Competitividad (TEC2013-47037-C05-5-R) and European regional funds (FEDER).

J. D. Ruiz, F. L. Martínez-Viviente and J. Hinojosa are with the Department of Electronics and Computer Engineering, A. Álvarez-Melcón is with the Department of Information and Communications Technology, Universidad Politécnica de Cartagena, Cartagena (Murcia) 30202, Spain (email: juan.hinojosa@upct.es).

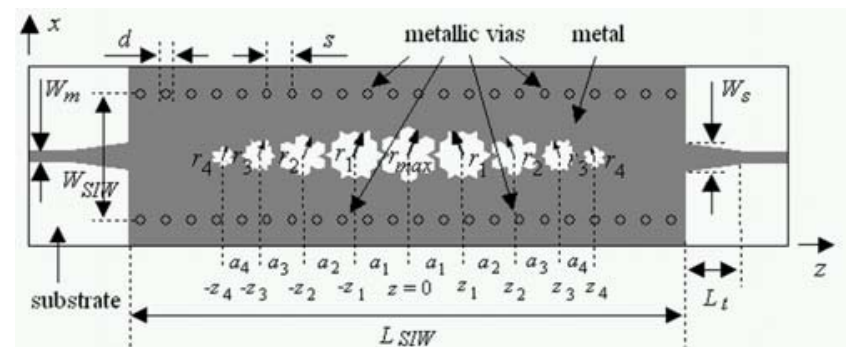

(a)

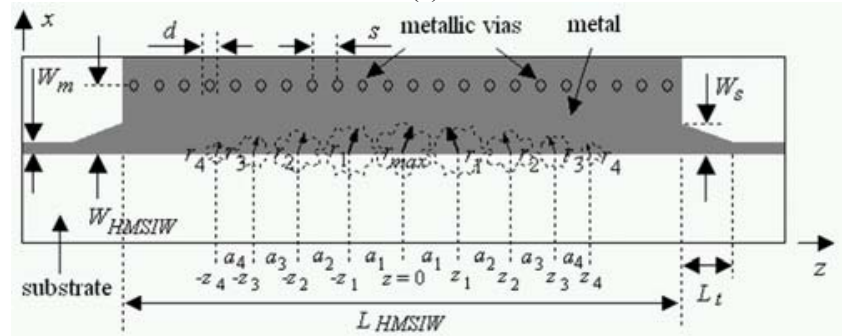

(b)

Fig. 1. Layouts of the proposed chirped and tapered (a) SIW-KFEBG and (b) HMSIW-KFEBG bandpass filters.

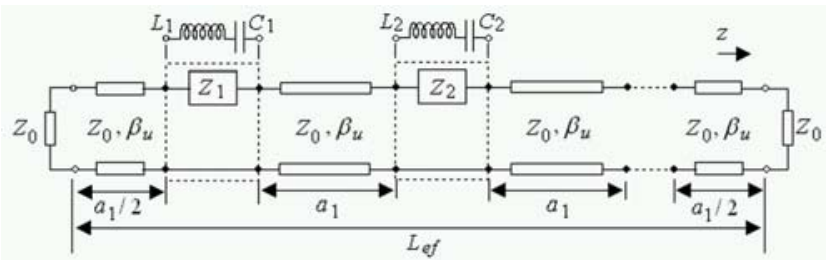

(a)

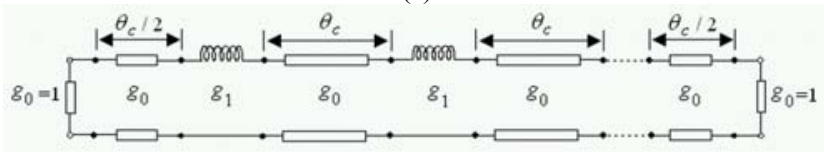

(b)

Fig. 2. (a) Equivalent circuit of a generalized bandpass periodic structure. (b) Generalized low-pass filter (LPF) prototype for periodic structures with lumped-distributed network.

\section{Filter Design APPROACH}

Using a previous work [6], the design approach starts by applying a Cauchy tapering function $T_{i}$ to the radii $r_{i}$ of each Koch fractal (KF), and a chirping period $a_{i}$ to the separation between Koch fractals (Fig. 1)

$$
\begin{gathered}
r_{i}=r_{\text {max }} T_{i} \quad i=0,1, \ldots \\
T_{i}=\left[1+(i / 2)^{2}\right]^{-1} \quad i=0,1, \ldots
\end{gathered}
$$


TABLE I

GEOMETRIC PARAMETERS OF THE SIW-KFEBG AND HMSIW-KFEBG

\begin{tabular}{|c|c|c|c|c|c|c|c|c|c|c|c|}
\hline Parameter & $\begin{array}{l}\text { Value } \\
(\mathrm{mm})\end{array}$ & Parameter & $\begin{array}{l}\text { Value } \\
(\mathrm{mm})\end{array}$ & Parameter & $\begin{array}{l}\text { Value } \\
(\mathrm{mm})\end{array}$ & Parameter & $\begin{array}{l}\text { Value } \\
(\mathrm{mm})\end{array}$ & Parameter & $\begin{array}{l}\text { Value } \\
(\mathrm{mm})\end{array}$ & Parameter & $\begin{array}{l}\text { Value } \\
(\mathrm{mm})\end{array}$ \\
\hline$W_{\text {HMSIW }}$ & 10 & $L_{S I W}$ & 65 & $W_{s}$ & 2.2 & $r_{1}$ & 4.441 & $r_{4}$ & 1.110 & $a_{3}$ & 6.107 \\
\hline$d$ & 1 & $L_{H M S I W}$ & 65 & $L_{t}$ & 11 & $r_{2}$ & 2.776 & $a_{1}$ & 11.103 & $a_{4}$ & 3.838 \\
\hline
\end{tabular}

$$
a_{i}=r_{i-1} / C \quad i=1,2, \ldots
$$

where $r_{i}$ and $r_{\max }$ are the $i$-th and maximum Koch fractal radii, respectively. $a_{i}$ preserves a constant $r / a$ ratio (denoted $C)$ between the centers of adjacent Koch fractals along the structure. In (3), $r_{i}$ is computed from (1), $r_{0}$ is equal to $r_{\text {max }}$, and $C$ is a constant $\geq 0.5$ in order to achieve a wide stop-band behavior [5], [6].

The proposed BPFs (Fig. 1) can be modeled by a transmission line of effective length $L_{e f}$ periodically loaded with $N_{e q}$ equivalent lumped impedances $Z_{i}$ as in Fig. 2(a) [7] using the transformation [8]

$$
L_{e f}=L \cdot \int_{-0.5}^{0.5} T(z / L) d(z / L)=L \cdot A_{e q} \text { and } N_{e q}=L_{e f} / a_{1}
$$

where $L=\sum_{i=1}^{(N+1) / 2} 2 \cdot a_{i}$ and $A_{e q}=0.57$ for the Cauchy tapering function (2). Note that, due to the tapering applied, the BPFs of Fig. 1 behave as BPFs of reduced order $N_{e q}<N$. The BPF equivalent circuit of Fig. 2(a) is assumed lossless and the coupling effects between resonators are neglected. $Z_{0}$ and $\beta_{u}$ are, respectively, the characteristic impedance and the phase constant of the unperturbed structure (without KFEBG). $a_{1}$ is the period defined in (3) for $i=1$ and a defined value $C$. Between two possible options for $Z_{i}$, we have chosen the simplest, which consists in considering the same series $L_{1} C_{1}$ resonant circuit for all the KFEBG patterns. In this way, we can introduce a design procedure based on the LPF prototype shown in Fig. 2(b) [9], with the characteristic impedance of all transmission lines scaled to the same value as the source impedance $g_{0}=1$. Due to the periodicity of the structure, each KFEBG pattern with $L_{1} C_{1}$ resonant circuit has the same $g_{1}$ and each transmission line section of length $a_{1}$ has the same phase shift $\theta_{c}$. The element values from lowpass to bandpass transformation are as follows:

$$
L_{1}=Z_{0}\left(\frac{1}{F B W \omega_{0}}\right) g_{1}, C_{1}=\frac{1}{\left(\omega_{0}\right)^{2} L_{1}} \text { and } a_{1}=\left.\frac{\theta_{C}}{\beta_{u}}\right|_{f_{2}}
$$

with $F B W=\left(f_{2}-f_{1}\right) / f_{0}$ and $f_{0}=\sqrt{f_{1} f_{2}}\left(f_{1}\right.$ and $f_{2}$ are the passband-edge frequencies). While $f_{2}$ depends on $a_{1}, f_{1}$ (lower cutoff frequency of the passband) is linked to the cutoff frequency $f_{c}$ of the SIW (HMSIW) which will be optimized during the design procedure $\left(f_{c}<f_{1}\right)$. The constant $C=r / a$ and the number $N$ of KFEBG patterns are respectively related with the width and the rejection level of the stopband [5].

According to given design specifications (bandwidth, rejection level, etc.) and a selected substrate, the design procedure of the proposed BPFs (Fig. 1) is as follows.

Step 1)Simulate the LPF prototype (Fig. 2(b)) and increase $N$ to get the desired stopband rejection. Obtain $g_{1}$ and $\theta_{C}$ by optimizing the LPF according to $3-\mathrm{dB}$ cutoff at the normalized angular frequency $(\omega=1 \mathrm{rad} / \mathrm{s})$ and for a desired stopband frequency $\left(\omega_{s}\right)$.

Step 2)Extract the geometric parameters of the SIW (HMSIW) waveguide using the analytical relationships in references [3], [4] for $f_{c}<f_{1}$.

Step 3)Optimize the transition microstrip to SIW (HMSIW) for $Z_{0}=50 \Omega$ using a full-wave simulator (HFSS). Extract transition dimensions, $\beta_{u}$ (effective permittivity $\varepsilon_{\text {ref }}$ ) and compute $L_{1}, C_{1}$ and $a_{1}$ from Step 1) and equation (5).

Step 4)Extract the geometric parameters of the chirped and tapered SIW (HMSIW)-KFEBG BPF using (1)-(4) and the previous data. Simulate the BPF circuit (Fig. 2(a)) with the obtained $N_{e q}$. If the desired stopband rejection and bandwidth are achieved then go to Step 5). If not, vary $f_{c}$ of the SIW (HMSIW) and then go back to Step 2).

Step 5)Simulate the chirped and tapered BPF using a fullwave simulator and the previous data.

Step 6)Final design: optimize basic dimensions of the previous BPF using a full-wave simulator to improve performance. Extract the final geometric parameters.

\section{EXPERIMENTAL RESULTS}

The proposed BPFs (Fig. 1) were designed with the above design procedure with $C=r / a=0.5$, an Arlon AD1000 substrate $\left(\varepsilon_{r}=10.2, \operatorname{tg} \delta=0.0023\right.$ at $10 \mathrm{GHz}$ and substrate thickness $h=0.635 \mathrm{~mm}$ ) and the following specifications: ripple $<1 \mathrm{~dB}$, insertion loss $I L<2 \mathrm{~dB}$, return loss $R L>10 \mathrm{~dB}$, stopband rejection level $S R L>35 \mathrm{~dB}$ at $f_{S}=5 \mathrm{GHz}$, a central frequency $f_{0}=3.4 \mathrm{GHz}$ and $3 \mathrm{~dB}$ cutoff bandwidth of $B W=$ $0.65 \mathrm{GHz}$ and $B W=0.85 \mathrm{GHz}$ for the SIW-KFEBG and HMSIW-KFEBG, respectively. Intermediate data obtained from the design procedure for both filters were: $g_{1}=1, N=9$, $\theta_{c}=114.74^{\circ}, \varepsilon_{r e f}=6.3$ and $N_{e q}=4$. Their geometric parameters, after the final optimization, are provided in Table I. These structures were fabricated using the PCB process and measured with a Rohde \& Schwarz ZVA network analyzer. 
TABLE II

COMPARISON BETWEEN THE PROPOSED FILTERS AND THE REFERENCES

\begin{tabular}{|c|c|c|c|c|c|c|c|}
\hline & 3-D size & FBW $(\%)$ & Ripple (dB) & $I L(\mathrm{~dB})$ & $R L(\mathrm{~dB})$ & Stopband bandwidth $(\mathrm{GHz})$ & $S R L$ \\
\hline Fig. 3(a) & $0.24 \lambda_{0} \times 0.008 \lambda_{0} \times 0.73 \lambda_{0}$ & 18.9 & 0.43 & 1.3 & 12.3 & 2.4 & $42 \mathrm{~dB} @ 5.6 \mathrm{GHz}$ \\
\hline Fig. $22[1]$ & $0.52 \lambda_{0} \times 0.02 \lambda_{0} \times 1.24 \lambda_{0}$ & 61.2 & 1.5 & 1.3 & 10 & - & $50 \mathrm{~dB} @ 20 \mathrm{GHz}$ \\
\hline Fig. 16 [2] & $0.29 \lambda_{0} \times 0.009 \lambda_{0} \times 0.75 \lambda_{0}$ & 74 & 1.4 & 0.5 & 9 & 0.45 & $50 \mathrm{~dB} @ 4 \mathrm{GHz}$ \\
\hline Fig. 5 [10] & $0.31 \lambda_{0} \times 0.035 \lambda_{0} \times 1.22 \lambda_{0}$ & 19.5 & 0.05 & 3.2 & 15 & 4 & 42dB@5.6GHz \\
\hline
\end{tabular}

where $\lambda_{0}$ is free-space wavelength. The sizes of [1] and [10] have been calculated for 9 cells. Any size includes the microstrip feeding.

Fig. 3 shows a good agreement between the EM simulation (HFSS) and measurement results for the proposed BPFs. The measured central frequency of the stopband $f_{s}=5.6 \mathrm{GHz}$ is slightly higher than the initial design specifications $(5 \mathrm{GHz})$, since the phase constant was considered constant and unperturbed $\left(\beta_{u}\right)$ in the equivalent circuit. The number of reflection zeroes must be equal to $N_{e q}=4$. However, the number of reflection zeroes in the frequency axis shown in Fig. 3(a) for the SIW-KFEBG does not match this value. This is because 2 reflection zeroes become complex due to weak couplings between resonators in the SIW-KFEBG. These couplings are stronger in the HMSIW-KFEBG (Fig. 3(b)) because the resonators are crossed by fringing fields. The central frequency is around $f_{0}=3.4 \mathrm{GHz}$ for both BPFs. The measured insertion losses for the SIW-KFEBG and HMSIWKFEBG are $1.3 \mathrm{~dB}$ and $1.7 \mathrm{~dB}$, respectively, which include the extra loss from the SMA connectors. The measured return losses are better than $11.4 \mathrm{~dB}$ for both BPFs. The stopband rejection is better than $20 \mathrm{~dB}$ up to the spurious band, which appears at a frequency higher than $6 \mathrm{GHz}$. Table II provides a performance comparison between the proposed BPFs and other SIW-EBG designs [1], [2], and also for a classical SIWChebyshev BPF [10]. The proposed BPFs have better performance than the other SIW-EBGs in size, ripple, return loss and stopband bandwidth, and in size and insertion loss with respect to the classical SIW-Chebyshev BPF.

\section{CONCLUSION}

A new design approach is applied to SIW and HMSIW BPFs implemented with KFEBG patterns etched on the waveguide surface, allowing a radius-to-period ratio $r / a \geq 0.5$. A chirped and tapered technique based on a Cauchy function is applied to the KFEBG patterns to improve the BPF responses. Performance of both BPFs are good as demonstrated by their measured insertion $(I L<1.7 \mathrm{~dB})$ and return $(R L>11.4 \mathrm{~dB})$ losses. A good stopband rejection $(S R L$ $>39 \mathrm{~dB}$ ) is obtained with nine chirped and tapered Koch fractal patterns.

\section{REFERENCES}

[1] Z-C. Hao, J-X. Chen, X-P. Chen, and K. Wu, "Compact super-wide bandpass substrate integrated waveguide (SIW) filters," IEEE Trans. Microw. Theory Tech., vol. 53, pp. 2968-2977, Sep. 2005.

[2] L-S. Wu, X-L. Zhou, W-Y. Yin, C-T. Liu, L. Zhou, J-F. Mao, and H-L. Peng, "A new type of periodically loaded half-mode substrate integrated waveguide and its applications," IEEE Trans. Microw. Theory Tech., vol. 58, pp. 882-893, Apr. 2010.

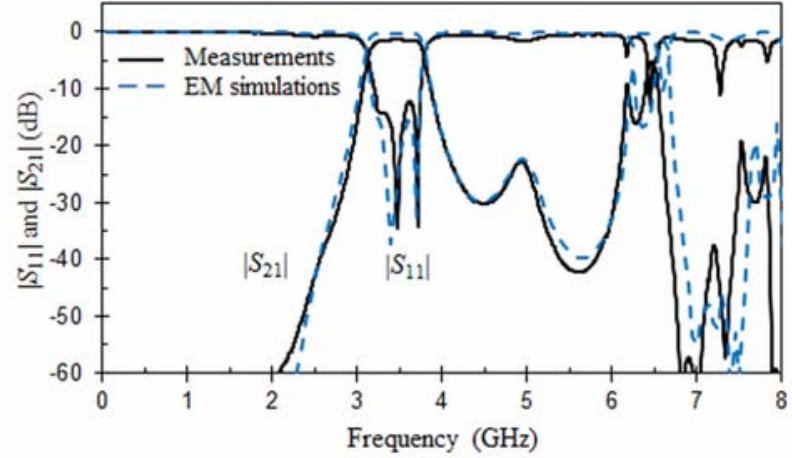

(a)

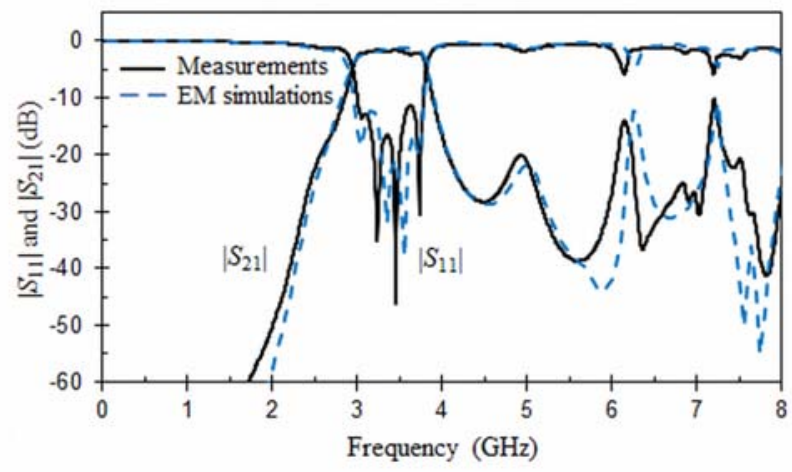

(b)

Fig. 3. EM simulation (dashed lines) and measured (solid lines) results for the (a) SIW-KFEBG and (b) HMSIW-KFEBG bandpass filters.

[3] F. Xu, and K. Wu, "Guided-wave and leakage characteristics of substrate integrated waveguide," IEEE Trans. Microw. Theory Tech., vol. 53, pp. 66-73, Jan. 2005.

[4] Q. Lai, C. Fumeaux, W. Hong, and R. Vahldieck, "Characterization of the propagation properties of the half-mode substrate integrated waveguide," IEEE Trans. Microw. Theory Tech., vol. 57, pp. 19962004, Aug. 2009

[5] J. D. Ruiz, F. L. Martínez, and J. Hinojosa, "Novel compact wide-band EBG structure based on tapered 1-D Koch fractal patterns," IEEE Antennas Wireless Propag. Lett., vol. 10, pp. 1104-1107, Oct. 2011.

[6] J. D. Ruiz, F. L. Martínez, and J. Hinojosa, "A flat-passband and widestopband low-pass filter based on tapered Cauchy microstrip Koch fractal EBG structure," in Proc. METAMATERIALS, 2014.

[7] M. Rahman and M. A. Stuchly, "Transmission line-periodic circuit representation of planar microwave photonic bandgap structures," Microw. Opt. Technol. Lett., vol. 30, pp. 15-19, Jul. 2001.

[8] M. A. G. Laso, M. J. Erro, T. Lopetegui, et al., "Optimization of tapered bragg reflectors in microstrip technology," Int. Infrared Millimet Waves, vol. 21, pp. 231-245, Feb. 2000.

[9] R. J. Cameron, C. M. Kudsia, and R. R. Mansour, Microwave filters for Communication systems. Hoboken, NJ, USA: Wiley, 2007, Ch. 12, pp. 431-467.

[10] Z-C Hao, W. Hong, H. Li, H. Zhang, and K. Wu, "A broadband substrate integrated waveguide (SIW) filter," in Proc. IEEE AP-S Int. Symp., Washington DC, vol. 1B, Jul. 2005, pp. 598-601. 